\title{
Zinner's Syndrome - The Value of Clinical Imaging and Morphopathological Findings for Diagnosis
}

\author{
Bogdan Călin Chibelean1, Octavian Sabin Tătaru², Angela Borda², Andrada Loghin1, Alin Nechifor- \\ Boilă1, Orsolya Mártha1 \\ 1 Urology Clinic, County Clinical Hospital, “George Emil Palade” University of Medicine and Pharmacy, Science and Technology, \\ Târgu Mureș, Romania \\ 2 Department of Histology, County Clinical Hospital, "George Emil Palade” University of Medicine and Pharmacy, Science and \\ Technology, Târgu Mureș, Romania
}

\section{CORRESPONDENCE \\ Octavian Sabin Tătaru \\ Str. Gheorghe Marinescu nr. 1 \\ 540103 Târgu Mureș, Romania \\ Tel: +40 265215133 \\ E-mail: sabin.tataru@yahoo.com}

\section{ARTICLE HISTORY}

Received: September 28, 2019

Accepted: December 17, 2019
Bogdan Călin Chibelean • Str. Gheorghe Marinescu nr. 1, 540103 Târgu Mureș, Romania, Tel: +40 265215 133, E-mail: calinchibelean@yahoo.com

Angela Borda • Str. Gheorghe Marinescu nr. 38 540139 Târgu Mureș, Romania. Tel: +40 265215 551, E-mail: aborda2001@gmail.com

Andrada Loghin • Str. Gheorghe Marinescu nr. 1 540103 Târgu Mureș, Romania. Tel: +40 265215133 E-mail: andradaloghin@yahoo.com

Alin Nechifor-Boilă • Str. Gheorghe Marinescu nr. 1, 540103 Târgu Mureș, Romania. Tel: +40 265215 133, E-mail: nechiforalin@yahoo.com

Orsolya Mártha • Str. Gheorghe Marinescu nr. 1, 540103 Târgu Mureș, Romania. Tel: +40 265215 133, E-mail: orsim@hotmail.com

\begin{abstract}
Introduction: Cystic congenital malformations of the seminal vesicle are unusual. More than half of them are associated with ipsilateral renal agenesis. This disease was first described by Zinner in 1914, and since then, more than 200 cases have been reported. Most of the patients with this congenital disease present few symptoms until the middle-age. Case presentation: $A$ 61-year-old male was referred to the Urology Clinic with intermittent pain and sensibility in his lower left abdomen in the last 12 months accompanied by intermittent bowel obstruction. The digital rectal examination could not assess the prostate due to a rectal stenosis. On computed tomography and magnetic resonance imaging (MRI) a cystic tumor with close relations with the bladder wall and prostate, associated with left kidney agenesis was identified. The cystic tumor was surgically removed. The histopathological examination of the tumor revealed a multilocular seminal vesicle cyst, with a muscular wall. Conclusions: Seminal vesicle cysts combined with ipsilateral renal agenesis are rare urological anomalies. Imaging allows a comprehensive investigation of renal and seminal vesicle anomalies. MRI seems to better visualize cysts and differentiate them from ureteroceles, while the histopathological examination contributes to a correct diagnosis. A multidisciplinary team (urologists, radiologists, and morphologists) is necessary for the proper diagnosis of Zinner's syndrome.
\end{abstract}

Keywords: Zinner's syndrome, renal agenesis, seminal vesicle cyst

\section{INTRODUCTION}

Zinner's syndrome is a urogenital birth defect comprised of enlargement of the seminal vesicle with ipsilateral renal agenesis. ${ }^{1}$ The first published data on the pathology belongs to Zinner in 1914. In Zinner's original report, he noted the frequent association of a seminal vesicle cyst with renal agenesis and also with other genital anomalies such as ejaculatory duct obstruction. ${ }^{2}$ In a review from 2009, Pereira et al. reported on 200 cases of seminal vesicle cysts associated with 
ipsilateral renal agenesis from the literature. ${ }^{3}$ Since then, several cases have been reported by other authors such as Slaoui et al. or Xiao-Song Jiang et al.1,4

Zinner's syndrome is a developmental embryologic anomaly that appears from the second until the third gestational month in the distal portion of the mesonephric or the Wolffian duct. ${ }^{5}$ We present the case of a patient whose first symptoms had occurred more than 15 years ago. Due to an incomplete diagnosis, the patient had been known with unilateral kidney agenesis (UKA). This article aims to emphasize the value of clinical, imaging, and morphological examination in the correct diagnosis and management of such cases.

\section{CASE PRESENTATION}

We present the case of a 61-year-old male, father of two children, who has been referred to our department presenting recurrent pain in his lower left abdomen for approximately one year, accompanied by symptoms of intermittent bowel obstruction with cramps and intermittent constipation, but with no history of fever, hematuria, pyuria, or hematospermia.

The patient's medical history revealed an open midline laparotomy performed fourteen years ago for a cystic pelvic tumor that caused a complete bowel obstruction. The cyst had been excised, but no histopathological report was available. At the time of the procedure, the patient had found out that he only had his right kidney, fact confirmed by the computed tomography (CT) scan performed at admission.

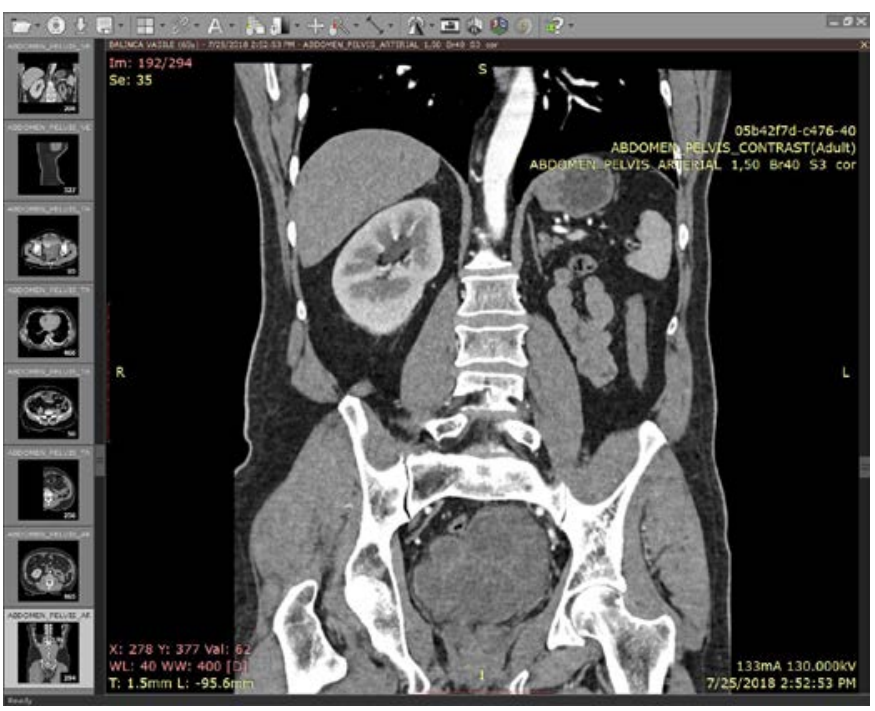

FIGURE 1. CT scan showing left renal agenesis and seminal vesicle cyst on arterial time
TABLE 1. Blood and urine analysis

\begin{tabular}{lc}
\hline Preoperative laboratory evaluations & Value \\
\hline Hemoglobin $(\mathrm{g} / \mathrm{dL})$ & 14.61 \\
Leukocytes $(\mu \mathrm{L})$ & 5.310 \\
Serum creatinine/urea $(\mathrm{mg} / \mathrm{dL})$ & $0.82 / 40$ \\
Creatinine clearance $\left(\mathrm{mL} / \mathrm{min} / 1.73 \mathrm{~m}^{2}\right)$ & 72.4 \\
Urine analysis & Leukocytes $(1-2$ cells/field) \\
Urine culture & $<1,000 \mathrm{CFU} / \mathrm{mL}$ \\
\hline
\end{tabular}

The examination showed a patient in good general condition, afebrile. The abdomen was sensitive at palpation of the left lower quadrant, but with no palpable mass. External genitalia and vas deferens were normal. Ultrasonography of the scrotum did not reveal any other genital anomalies. Transrectal ultrasonography could not be performed due to a bulging mass in the rectum. Laboratory tests, urinalysis, urine culture, quantitative prostate specific antigen (PSA) were in normal range $(2.44 \mathrm{ng} / \mathrm{mL}$ ) (Table 1$)$. The digital rectal examination revealed a mass bulging into the rectum, with the impossibility to perform a prostate examination. On abdominal and pelvic computed tomography (CT) scan (Figure 1) and magnetic resonance imaging (MRI) (Figure 2), a cystic tumor of $10 \times 9.6 \times 12 \mathrm{~cm}$, adherent to the posterior bladder wall, prostate, and sacral bone, associated with left kidney agenesis was found. The patient has two children and did not want any other offspring, and the semen analysis was not routinely performed. Given the existence of an ectopic left kidney, an open surgery approach was decided for the removal of the cystic tumor. On histopathological examination, the tumor

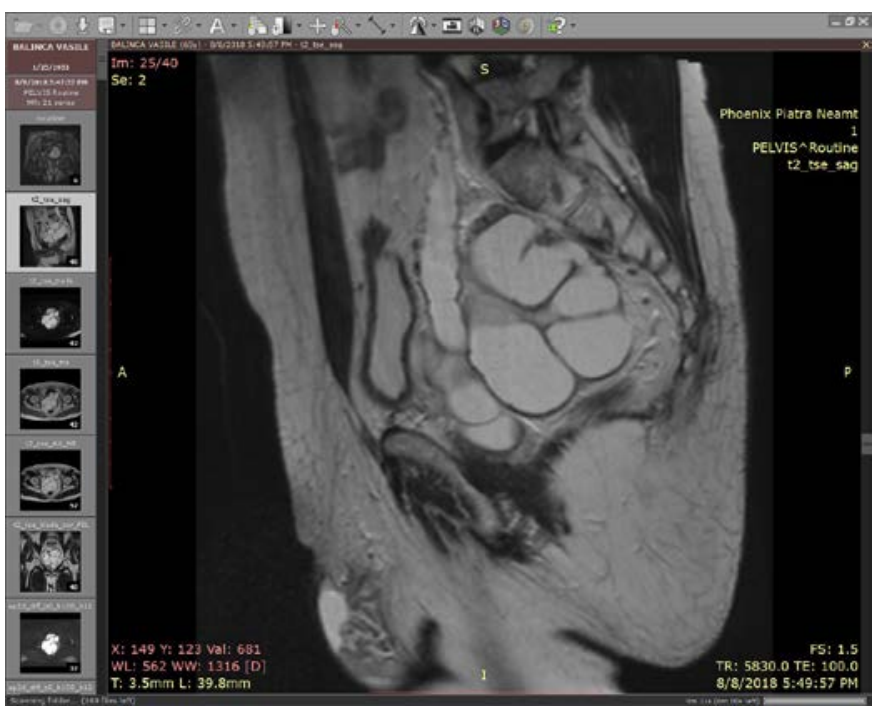

FIGURE 2. Sagittal T2-W MRI showing dilated ureter and seminal vesicle with multiple cysts 


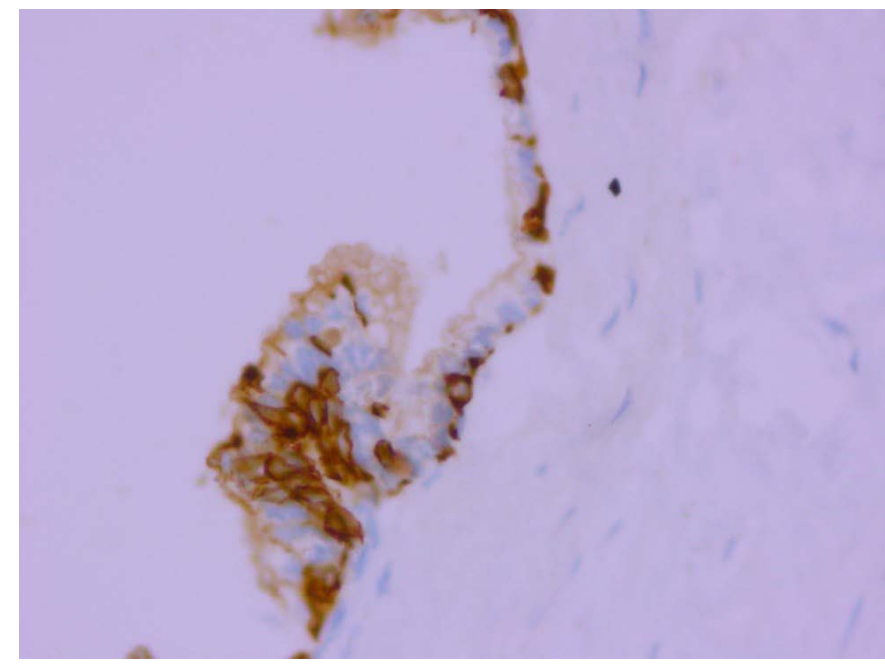

FIGURE 3. Histopathological aspect of the excised cyst: low molecular weight cytokeratin 34 beta E12

was shown to be a multilocular seminal vesicle cyst, with a muscular wall and an epithelium composed of columnar and basal cells, with lipofuscin pigment in the cytoplasm and nuclei with marked atypia. The epithelial component was positive for PANCK (AE1/AE3) and negative for PSA (Figures 3, 4, and 5).

The patient agreed to the publication of his data, and the institution where the patient had been admitted approved the publication of the case.

\section{DISCUSSIONS}

The first case of hydrocele of the seminal vesicle was reported in 1872, by Smith. Zinner's syndrome, which associates

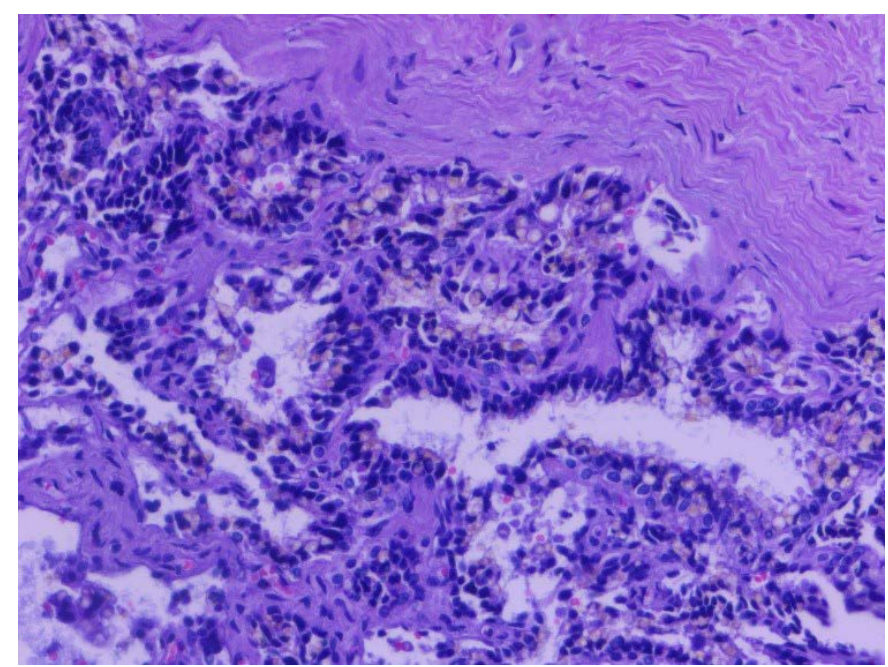

FIGURE 5. Histopathological aspect of the excised cyst: HE 40×, muscular wall and epithelium mucosa with lipofuscin in the secretory cells

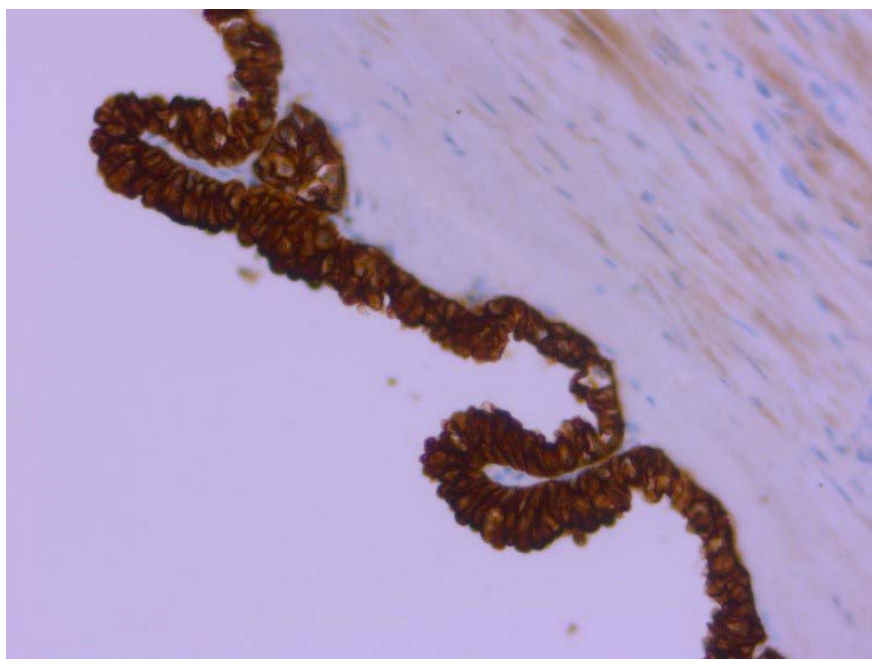

FIGURE 4. Histopathological aspect of the excised cyst: Epithelial component positive for PANCK (AE1/AE3)

UKA and ipsilateral seminal vesicle cyst, was first described in 1914.2,6 This embryogenesis anomaly is uncommon, and its actual prevalence is difficult to be identified. In 1990, an article published by Sheih et al. found 13 reports of cystic pelvic dilatations along with ipsilateral renal agenesis or dysplasia during ultrasound screening of 280,000 children in Taiwan, yielding a frequency of $0.00214 \%$ of Zinner's syndrome. ${ }^{7}$ In an article on unilateral kidney agenesis in newborns, with data derived from two birth defect registries, the authors looked at 447,885 births and identified 177 patients with UKA, with a prevalence at birth of $4.0 / 10,000$, but with no reported association of seminal vesicle anomalies; thus, we can state that it is indeed a rare entity. ${ }^{8}$ Although a few cases of Zinner's syndrome have been identified, UKA is present in $0.1 \%$ of newborns, and physicians should look for other genital and urinary anomalies that are present in up to $40 \%$ of patients. ${ }^{9}$ The size of the cysts can lead to early diagnosis due to complications that translate into symptoms. Small cysts $<5 \mathrm{~cm}$ are usually asymptomatic, but if larger than $12 \mathrm{~cm}$ in diameter, they may cause symptoms leading to obstruction of the bladder neck and lower digestive tract. ${ }^{10-13}$ Ultrasonography has been used for a long time in male patients suspected of congenital anomalies of the kidneys and urinary tract, being the imaging method of choice. However, lately CT and MRI scans have proven to be of use in determining if the cysts are associated with other urogenital anomalies such as ipsilateral renal agenesis. They also provide additional help in avoiding misdiagnosis and the necessary imaging information to properly manage the case. ${ }^{4,14,15}$ In retrospect, vasovesiculography was used in the past as part of the investigational tools for male infertility problems and 
to diagnose the dilatation of the seminal vesicle, but today it is not routinely used. ${ }^{3}$ Currently, there are no studies on large cohorts of patients regarding this diagnosis, as there were just over 200 cases reported until 2009. ${ }^{3}$

Embryologic anomalies develop in the first trimester of pregnancy, between the 4th and the 13th gestational week, in the caudal part of the mesonephric duct, which is attached to the urogenital sinus together with the ureteric bud. ${ }^{16}$ Early migration of the ureteric bud will affect the mesonephros, which will not develop properly and disappear. The outcome is kidney agenesis, together with poor development of the ejaculatory duct. ${ }^{16,17}$ The insufficient flow of seminal fluid due to poor drainage will cause distension of the seminal vesicle, leading to a cystic structure. ${ }^{18}$ Patient complaints are mainly related to filling or voiding bladder symptoms, including dysuria, polyuria, painless hematuria; symptoms related to cyst enlargement such as perineal pain, or obstructive mesonephric duct structures leading to epididymitis, pain following ejaculation, infertility, impaired defecation, and pain in the lower abdomen. ${ }^{16}$ Infertility is an issue in such patients, with oligospermia, oligoasthenozoospermia, and azoospermia as leading characteristics on semen analysis. ${ }^{16}$

Digital rectal examination identifies a large majority of seminal vesicle cysts. Intravenous urography shows an image of the urinary collecting system, providing important information related to a potential unilateral renal agenesis. Endorectal ultrasound is a rapid, nonionizing method of assessing the pelvic cystic structures, which may show an anechoic mass, surrounded by an enlarged wall. ${ }^{19} \mathrm{CT}$ scanning can provide very important information regarding the internal organs. One missing organ or an undefined modification of an organ, such as the seminal vesicle, can be precisely shown by the CT scan. There are CT signs that lead to a suspicion of a congenital abnormality, such as thick walls, a heterogeneous structure with irregular margins, and, sometimes, signs of an invasive mass. ${ }^{20,21}$ MRI can provide more information regarding the relations between pelvic organs, such as the seminal vesicles in male patients, can confirm that the cystic structures are in the seminal vesicles, and can also identify ectopic ureteric orifices. The examination seems to provide more detailed information compared to a CT scan, because of the multiplanar reconstruction. ${ }^{15}$ Fluid in the cysts that are hyperintense on T1-W images can be differentiated from fluid within ureteroceles and simple cysts that appear hypointense on $\mathrm{T} 1-\mathrm{W}$ pictures, as well as cystic masses in the seminal vesicle appearing hyperintense on $\mathrm{T} 2-\mathrm{W}$ pictures. $^{22,15}$ The differential diagnosis of cystic pelvic masses includes Mül- lerian duct cyst, prostatic cyst, diverticulosis of the vas deferens, leiomyoma of the bladder wall with degenerative changes, malignant lesions of the rectum with bladder involvement, ectopic ureterocele, and abscesses. ${ }^{16,23}$

On microscopy, the tumor was shown to be a multilocular seminal vesicle cyst, with a muscular wall and an epithelium composed of columnar and basal cells, with lipofuscin pigment in the cytoplasm and nuclei with marked atypia. The epithelial component was positive for PANCK (AE1/AE3) and negative for PSA. Small cysts are treated conservatively; surgical excision of a seminal vesicle cyst depends on the dimensions and localization of the cyst that causes symptoms. Laparoscopic and robotic techniques have replaced the open surgical approach. ${ }^{23}$ Because the laparoscopic approach was not available, we performed open surgery.

\section{CONCLUSIONS}

Seminal vesicle cysts combined with ipsilateral renal agenesis are unusual urological anomalies. CT and MRI allow a comprehensive investigation of renal and seminal vesicle anomalies. MRI seems to be superior in visualizing cysts and differentiating them from ureteroceles, while the histopathological examination contributes to a correct and definitive diagnosis. Its low incidence needs a multidisciplinary team, comprised of urologists, radiologists, and morphologists, to allow the proper diagnosis of Zinner's syndrome.

\section{COMPETING INTERESTS}

The authors declare that they have no conflict of interests and no financial interests related to the material in the manuscript.

\section{CONSENT}

Written informed consent was obtained from the patient for the publication of this report and any accompanying images. A copy of the written consent is available for review by the Editor-in-chief of this Journal.

\section{REFERENCES}

1. Slaoui A, Regragui S, Lasri A, Karmouni T, El Khader K, Koutani A, et al Zinner's syndrome: report of two cases and review of the literature. Basic Clin Androl. 2016;26:10.

2. Zinner A. Ein fall von intravesikaler Samenblasenzyste. Wien Med Wochenschr. 1914;64:604-609.

3. Pereira BJ, Sousa L, Azinhais P, et al. Zinner's syndrome: an up-to-date review of the literature based on a clinical case. Andrologia. 2009;41:322-330. 
4. Jiang X-S, Wang H-J, Lin J-H, et al. Zinner's syndrome: clinical features and imaging diagnosis. Asian J Androl. 2018;20:316-317,

5. Livingston $L$, Larsen $C R$. Seminal vesicle cyst with ipsilateral renal agenesis. AJR Am J Roentgenol. 2000;175:177-180.

6. Smith NR. Hydrocele of the seminal vesicle. Lancet. 1872;2:558.

7. Sheih CP, Hung CS, Wei CF, Lin CY. Cystic dilatations within the pelvis in patients with ipsilateral renal agenesis or dysplasia. J Urol. 1990;144:324327.

8. Laurichesse Delmas $\mathrm{H}$, Kohler $\mathrm{M}$, et al. Congenital unilateral renal agenesis: Prevalence, prenatal diagnosis, associated anomalies. Data from two birth-defect registries. Birth Defects Res. 2017;109:1204-1211.

9. Shariat SF, Naderi ASA, Miles B, Slawin KM. Anomalies of the wolffian duct derivatives encountered at radical prostatectomy. Rev Urol. 2005; 7:75-80.

10. Heaney JA, Pfister RC, Meares EM. Giant cyst of the seminal vesicle with renal agenesis. AJR Am J Roentgenol. 1987;149:139-140.

11. 1Arora SS, Breiman RS, Webb EM, Westphalen AC, Yeh BM, Coakley FV. $\mathrm{CT}$ and MRI of congenital anomalies of the seminal vesicles. AJR Am J Roentgenol. 2007;189:130-135.

12. Shah S, Patel R, Sinha R, Harris M. Zinner syndrome: an unusual cause of bladder outflow obstruction. BJR Case Rep. 2017:3:20160094.

13. Maehana T, Fukuta F, Kobayashi K, Hirobe M, Tanaka T, Masumori N Laparoscopic Surgery for Seminal Vesicle Cysts and Ureterocele with Urination Disorder: A Case Report of Zinner Syndrome. J Endourol Case Rep. 2018:4:35-38.

14. Chen H-W, Huang S-C, Li Y-W, Chen S-J, Sheih C-P. Magnetic resonance imaging of seminal vesicle cyst associated with ipsilateral urinary anomalies. J Formos Med Assoc Taiwan Yi Zhi. 2006;105:125-131.
15. Mehra S, Ranjan R, Garga UC. Zinner syndrome-a rare developmental anomaly of the mesonephric duct diagnosed on magnetic resonance imaging. Radio/ Case Rep. 2016;11:313-317.

16. van den Ouden D, Blom JH, Bangma C, de Spiegeleer AH. Diagnosis and management of seminal vesicle cysts associated with ipsilateral rena agenesis: a pooled analysis of 52 cases. Eur Urol. 1998;33:433-440.

17. Manousakas T, Kyriakou G, Serafetinides E, Giannopoulou M, Kyroudi A, Giannopoulos A. Partial vesiculectomy in an infertile man with semina vesicle cyst, ipsilateral renal agenesis, and cryptorchidism. Urology. 2002;59:602

18. Casey RG, Stunell H, Buckley O, Flynn R, Torreggiani WC. A unique radiological pentad of mesonephric duct abnormalities in a young man presenting with testicular swelling. Br J Radiol. 2008;81:e93-e96.

19. Narlawar RS, Hanchate V, Raut A, Hira P, Nagar A, Chaubal NG. Renal agenesis and seminal vesicle cyst. J Ultrasound Med Off J Am Inst Ultrasound Med. 2003;22:225-228.

20. Kenney PJ, Leeson MD. Congenital anomalies of the seminal vesicles: spectrum of computed tomographic findings. Radiology. 1983;149:247251

21. Fiaschetti V, Greco L, Giuricin V, et al. Zinner syndrome diagnosed by magnetic resonance imaging and computed tomography: role of imaging to identify and evaluate the uncommon variation in development of the male genital tract. Radiol Case Rep. 2017:12:54-58.

22. Murphy JO, Power RE, Akhtar M, Torreggiani WC, McDermott TED, Thornhill JA. Magnetic resonance imaging in the diagnosis of seminal vesicle cysts and associated anomalies. J Urol. 2003;170:2386.

23. Sundar R, Sundar G. Zinner syndrome: an uncommon cause of painful ejaculation. BMJ Case Rep. 2015;2015. 